\title{
Editorial
}

\section{Intelligent Control in Discrete Time for Autonomous Systems}

\author{
Chenguang Yang, ${ }^{1} \mathrm{Bin} \mathrm{Xu}^{2}{ }^{2}$ Hongbin $\mathrm{Ma},{ }^{3}$ Deqing Huang, ${ }^{4}$ and Jing $\mathrm{Na}^{5}$ \\ ${ }^{1}$ Zienkiewicz Centre for Computational Engineering, Swansea University, Swansea SA1 8EN, UK \\ ${ }^{2}$ School of Automation, Northwestern Polytechnical University, Xi'an 710072, China \\ ${ }^{3}$ School of Automation, Beijing Institute of Technology, Beijing 100081, China \\ ${ }^{4}$ School of Electrical Engineering, Southwest Jiaotong University, Chengdu 610031, China \\ ${ }^{5}$ Department of Mechanical Engineering, University of Bristol, Bristol BS8 1TR, UK \\ Correspondence should be addressed to Chenguang Yang; cyang@theiet.org
}

Received 17 May 2016; Accepted 17 May 2016

Copyright (C) 2016 Chenguang Yang et al. This is an open access article distributed under the Creative Commons Attribution License, which permits unrestricted use, distribution, and reproduction in any medium, provided the original work is properly cited.

The application of automatic systems is playing an increasingly important role in our modern society. Controllers as the soul of the autonomous systems are typically implemented by digital computers nowadays. In digital implementation, measurements are made and control actions are taken at discrete-time instants. In addition to digitalization, intelligence is another trend for modern control design, because the next generation of autonomous systems are required to perform tasks with smart and flexible strategic decisions. In this special issue, we bring together a number of works under the topic of autonomous discrete-time systems with intelligent controller. The contributors have investigated various applications of a wide range of autonomous systems, such as hypersonic missiles, robots, and underwater devices, and have also performed fundamental theoretical studies.

In the field of robotics, P. Liang et al. proposed an augmented approach for robot to understand human motion behaviours based on human kinematics and human postural impedance adaptation. A geometry vector approach was employed to extract human arm pose for stiffness transformation from Cartesian space to joint space. In another work, P. Liang et al. studied human limb stiffness estimation based on muscle electromyography (EMG) signals and applied the extracted limb stiffness onto control of a robot arm. Effectiveness has been tested and verified through disturbance rejection experiment. G. Huo et al. presented a PWMN-based scan matching approach for robot posture estimation, and it results in a faster convergence rate and shorter runtime with large moving steps and performs better for different footprints series, in comparison to PSM and Mb-ICP.

The problem of guidance and control of missile systems has attracted a large number of researchers in control and automation communities for decades. In this special issue, R. Sun and C. Ming presented a novel coning motion based guidance method for the missile's velocity control system. They used nonlinear programming method to present velocity profile and applied the dynamic inversion theory to design the velocity control system parameters. W. Fu et al. applied the sliding mode control (SMC) method and adaptive neural network (NN) technique to design controller for hypersonic missile. The flight path angle was used as the guidance command and the $\mathrm{NN}$ is used to approximate the uncertainty. Y. H. Fan et al. used discrete variable structure control (DVSC) with sliding vector to solve the control problem in the velocity and acceleration command tracking for a hypersonic cruise missile where the unknown dynamics and parameter disturbance were considered to improve the tracking performance.

Underwater vehicles and devices are playing important roles in deep sea exploration. Z. Mao and S. Yan focused on the problem of heat dissipation characteristics of the lithium battery inside autonomous underwater vehicles (AUVs). Based on the energy conservation equation, they have developed a model for the heat transfer process to analyse its characteristics. Y. Gou and W. Qi studied the positioning and tracking of underwater moving target by double-station array 
buoy, and unscented Kalman filter (UKF) theory was used to analyse the performance of the experiments. Y. Yu et al. made an effort to solve the control packets collision problem that exists widely in multichannel MAC protocol underwater, and soft time frequency masking was used to deal with the control packets collision. D. Teng and N. Zhu investigated the improvement of the performance of underwater transducer in its operating frequency, bandwidth, and miniaturization. They have developed a novel hybrid transducer based on the theory of coupled multimode vibrations and the method of FEM.

Various other applications have been reported in this special issue. L. Yu et al. proposed a faster and efficient learning scheme which originates from the thoughts of social organization, and the idea was formulated as methods for hypersonic flight control. Z. Mao et al. studied the power generation control system for a new drag-type vertical axis turbine with several retractable blades. L. Zhang and Y. Lyu presented distributed consensus filtering for a class of large-scale distributed systems with unknown boundary conditions. L. Song et al. adopted the $H \infty$ filter in the transfer alignment (TA) scheme, which was then used for the initial alignment of Inertial Navigation System (INS) on dynamical base. Q. Huang et al. simulated the wettability of hydrophobic surfaces and the dynamic behaviours of droplets impacting the hydrophobic surfaces.

The practical applications of automatic systems designed in discrete time are underpinned by the theoretical advances in the relevant topics. In this special issue, Y. Jiang et al. presented a thorough literature review on neural networks and fuzzy logic based control design technique developed in discrete time and their applications. B. Wu et al. worked on the control problem of the uncertain discrete-time-delay systems with disturbance and nonlinearity. They have designed a bounded controller based on state estimator and disturbance observer. K. Wang et al. introduced the idea of multiple models into adaptive dynamic programming (ADP), such that the consequent controller is able to deal with jumping parameters and to improve the transient response. X. Chen et al. focused on graph theory and presented a new theorem that if $G$ is a $k$-connected $(k \geq 2)$ almost claw-free graph of order $n$, then $G$ contains a spanning 3-ended tree. H. Sun et al. developed a novel composite adaptive antidisturbance controller for a class of discrete-time switched systems, such that good antidisturbance performance can be achieved.

The selected papers in the special issue could not cover all the recent advances of discrete-time intelligent systems, yet they present the state-of-the-art progress in this area. We hope these valuable papers may enrich knowledge in the discrete-time control community and provide guidance to the readers who are interested in this topic.

\section{Acknowledgments}

The guest editors would like to mention their appreciation to both the authors and the reviewers for their contribution towards the success of this special issue. The support by the Engineering and Physical Sciences Research Council (EPSRC) under Grant EP/L026856/2 is also appreciated.

Chenguang Yang
Bin Xu
Hongbin $\mathrm{Ma}$
Deqing Huang
Jing Na 


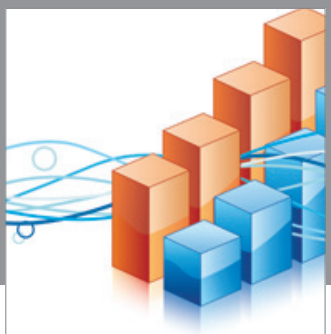

Advances in

Operations Research

vatem alat4

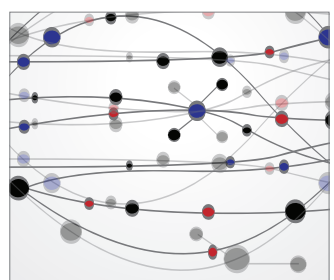

\section{The Scientific} World Journal
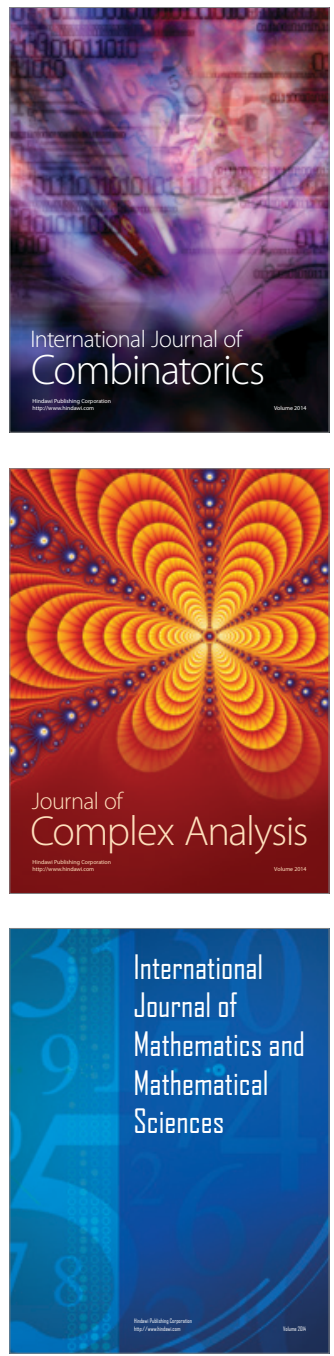
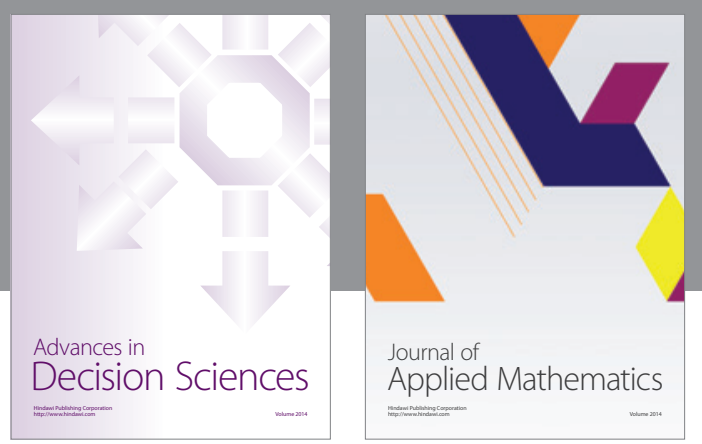

Algebra

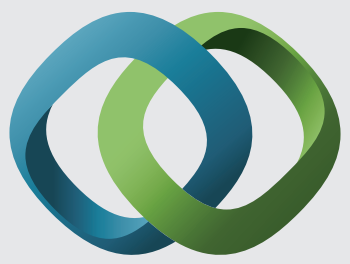

\section{Hindawi}

Submit your manuscripts at

http://www.hindawi.com
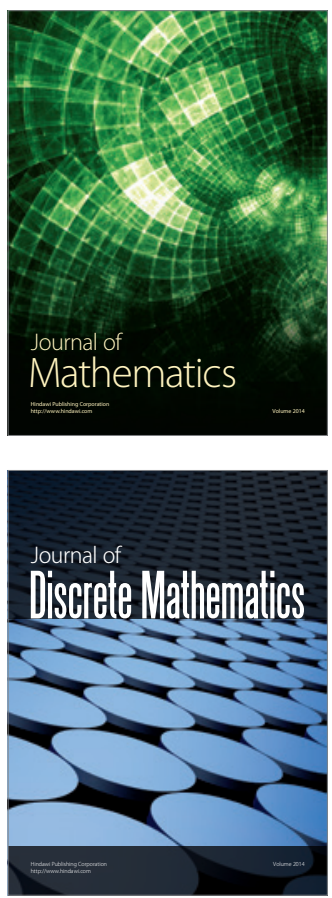

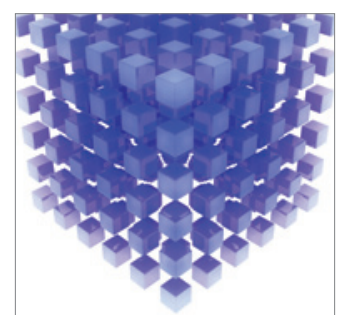

Mathematical Problems in Engineering
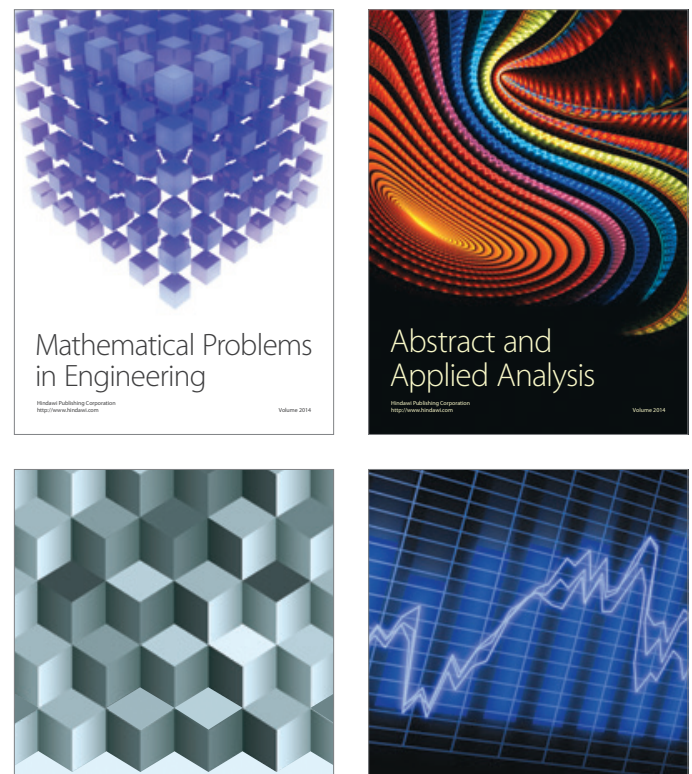

Journal of

Function Spaces

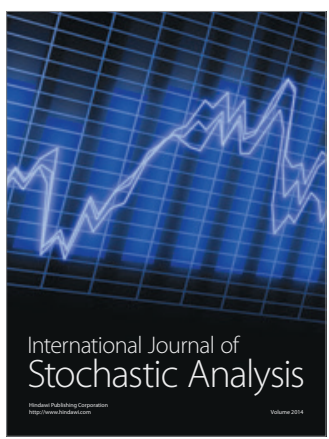

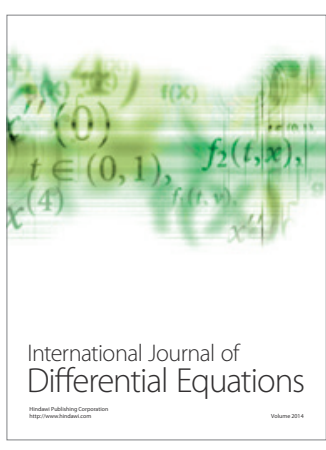
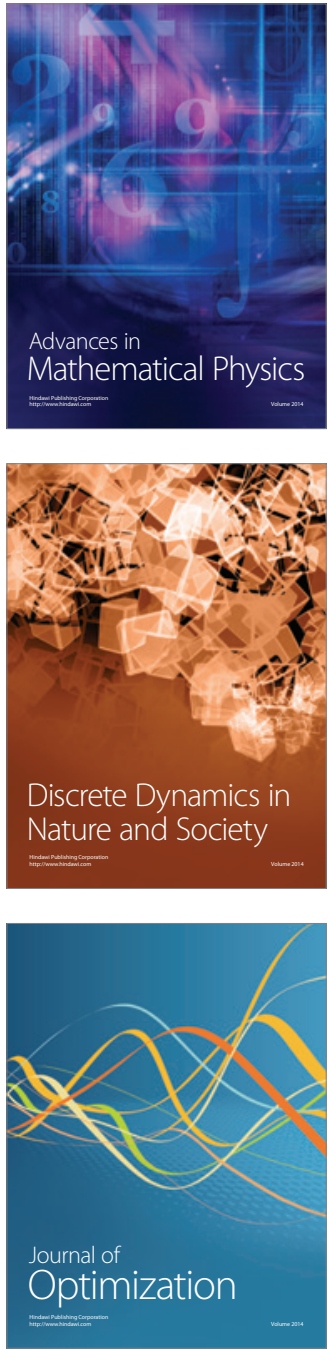